\title{
Gaussian Markov Random Fields-Based Features for Volumetric Texture Segmentation
}

\author{
Yasseen Almakady*, Sasan Mahmoodi*, Michael Bennett ${ }^{\dagger}$ \\ *Electronics and Computer Science, University of Southampton, United Kingdom \\ ${ }^{\dagger}$ Southampton NIHR Respiratory and Critical Care Biomedical Research Center, \\ University Hospital Southampton NHS Foundation Trust, United Kingdom \\ Email: $\{$ yham1n15,sm3y07,michael.bennett $\} @$ soton.ac.uk
}

\begin{abstract}
A new method based on three dimensional Gaussian Markov Random fields (3D-GMRF) is proposed in this paper for volumetric texture segmentation (VTS). A feature vector is extracted for each voxel in a given volumetric texture image. These feature vectors that consist of the estimated parameters of the GMRF and form the parameter volume are employed to segment volumetric textures. To overcome the issues related to boundaries and isolated voxels, a solution is proposed by sliding an averaging volume inside the parameter volume to assign each voxel a new feature vector derived as the mean of the surrounding voxels that are collected by the averaging volume. Our proposed method is evaluated on a synthetic volumetric texture and compared with another method demonstrating good segmentation performance. A further evaluation is carried out to examine the performance of the method proposed here in the presence of noise to show robustness to noise.
\end{abstract}

Keywords-3D-GMRF; Volumetric Texture; segmentation.

\section{INTRODUCTION}

The importance of cues provided by texture to human vision leads to the development of various methods for texture analysis. Texture has been widely employed in many applications to deliver texture-based solutions in many fields like in biomedical imaging, remote sensing, document processing and material inspection [6], [14]. Most texture analysis methods were proposed for analyzing texture acquired from two dimensional images (2D). However, with the availability of techniques that provide three-dimensional images (3D) such as Magnetic resonance imaging (MRI) and High-resolution computed tomography (HRCT) systems, the analysis of this kind of images using 2D texture methods could not deliver better solutions compared with 3D methods [4]. Volumetric texture ( or solid texture [11]) is the texture that can be found in 3D images and indexed by $(x, y, z)$ $\in \mathbb{R}^{3}$. This type of texture is commonly found in biomedical fields in which 3D data acquisition techniques provide rich information of the internal structure of objects [6]. Other fields also benefit from volumetric textures such as material analysis [8] and geoscience [13]. In this paper a method based on three dimensional Gaussian Markov Random fields (3D-GMRF) is proposed to segment textures found in 3D images. The reminder of the paper is organized as follows: in Section II, the existing volumetric segmentation methods are described. Our proposed method of volumetric texture segmentation (VTS) is introduced in Section III . In section IV the results are presented and discussed where Section V concludes the paper.

\section{RELATED WORK}

Image segmentation concerns partitioning images into homogeneous regions and it is a vital topic in image processing and computer vision fields. Texture is one of various features like color and shape that can be employed to segment images. While many methods were developed for 2D texture segmentation, little attention has been dedicated to volumetric texture segmentation [10] due to computational challenges involving an additional dimension [3]. Extracting 2D textures from slices of volumetric images is a common approach but does not fully benefit from the valuable information contained within volumetric data [6], [10]. Less attention has been devoted to volumetric texture segmentation in the literature. Most works on volumetric texture analysis were developed for application to medical image analysis. A method for volumetric texture segmentation based on Fourier domain filtering known as a Multiresolution Volumetric Texture Segmentation (M-VTS) is presented in [3]. In this method, textural measurements are extracted from the Fourier domain of the volumetric image and then a multiresolution classification algorithm is used to classify voxels before boundary refinement is performed. Another novel work is presented in [10] where a fully automated segmentation is proposed to segment prostatic adenocarcinoma in high resolution MRI data. In [2], Wavelet-based support vector machines (W-SVMs) are trained to capture kidney texture in three-dimensional (3D) MR images to automatically segment the kidney. Using extended 3D grey-level co-occurrence matrices (GLCM), the method presented in [9] extracts features from these matrices and uses them to segment the volumetric texture based on octree structure. Local binary patterns (LBP) are used in [1] to extract texture features from orthogonal planes of 3D MRI images and utilized for the detection of brain tumor. 


\section{Volumetric Texture Segmentation (VTS)}

\section{A. $3 D-G M R F$}

GMRF has been used for modeling image texture where model parameters are employed as features and used for texture classification [4], [5] and segmentation [7]. In our work presented in [4], an extension to 3D-GMRF is proposed where the estimated model parameters are successfully utilized as features and used for volumetric texture classification.

Let $\Omega=\{v=(i, j, k) \mid 1 \leq i \leq H, 1 \leq j \leq W, 1 \leq k \leq D\}$ denote the set of voxels indexed by $(i, j, k)$ on a $H \times W \times D$ $3 \mathrm{D}$ lattice corresponding to voxels in a $3 \mathrm{D}$ image volume. The local conditional probability density function of the intensity value $g_{v}$ at location $v$ is defined by:

$p\left(g_{v} \mid y_{v+r}, r \in N\right)=\frac{1}{\sqrt{2 \pi \sigma^{2}}} \exp \left\{-\frac{1}{2 \sigma^{2}}\left(g_{v}-\lambda-\sum_{r \in N} \alpha_{r}\left(y_{v+r}-\lambda\right)\right)^{2}\right\}$

where $N$ is the neighborhood of the voxel at location $v$, $\alpha_{r}$ are the interaction parameters that measure the influence on a voxel by neighbors' intensity values $y_{v+r}$ located at a relative position $r$ [4], [12] and $\lambda$ is the mean of the voxels in the neighborhood $N$. The neighborhood scheme adopted here is a cube with size $n \times n \times n$ which is used to collect observations. The unknown parameters $\alpha_{r}, \sigma^{2}$ and $\lambda$ need to be calculated to solve equation (1). The parameters $\alpha_{r}$ and $\sigma^{2}$ can be estimated using the Maximum Likelihood Estimation (MLE) [15] while $\lambda$ is calculated directly by taking the mean of the processed region to avoid dealing with non-linear equations system which are expensive to solve and could suffer from instability [4]. After applying MLE to estimate parameters $\alpha_{r}$ and $\sigma^{2}$ by taking the partial derivative of the log-likelihood function with respect to $\alpha_{r}$ and $\sigma^{2}$ and setting them to zero; the following solutions are obtained [4]:

$$
\begin{gathered}
\alpha_{v}=\left(\sum_{v \in \Omega_{v}} y_{v} y_{v}^{T}+c I\right)^{-1}\left(\sum_{v \in \Omega_{v}} y_{v} g_{v}\right) \\
\sigma_{v}^{2}=\frac{1}{\left|\Omega_{v}\right|} \sum_{v \in \Omega_{v}}\left(g_{v}-\alpha_{v} y_{v}\right)^{2}
\end{gathered}
$$

Where $\alpha_{v}=\operatorname{row}\left[\alpha_{r}\right]$ and $y_{v}=\operatorname{col}\left[y_{v+r}\right]$ for $r \in N, T$ is the transpose operation, $\Omega_{v}$ is the estimation cube which will be explained in Section III-B, $I$ is an identity matrix and $c$ is a regularization parameter to ensure that $y_{v} y_{v}^{T}$ matrix is invertible (nonsingular) to find solutions [16].

\section{B. Feature extraction}

The calculated parameters $\alpha_{v}, \sigma_{v}^{2}$ and $\lambda_{v}$ are the components of the feature vector and contain information about the local texture. This feature vector is extracted for each voxel at location $v$ rather than for the entire volume resulting in:

$$
f_{v}=\left\{\alpha_{v}, \sigma_{v}^{2}, \lambda_{v}\right\}
$$

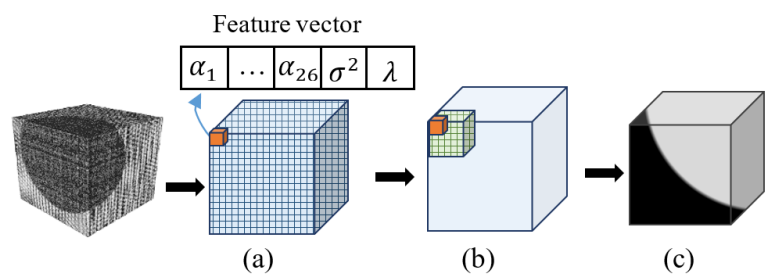

Figure 1. Overview of VTS : (a) The GMRF parameters are estimated to construct a feature vector at each voxel which results in a parameter volume. (b) the parameter volume is refined by moving an averaging volume. (c) the segmentation is performed using $k$-mean clustering algorithm.

To this end, instead of sliding the neighborhood cube, that used to collect observations, inside the entire volume; the neighborhood cube is slid inside a smaller volume called estimation cube $\Omega_{v}$ [4]. The estimation cube is also moving inside the volumetric image to cover the entire volume. As the cubic neighborhood system is adopted in this paper with dimensions $n \times n \times n$ and $n=3$, therefore 26 voxels are involved in the neighborhood system leading to the same length of the estimated parameter $\alpha_{v}$ vector. Hence, in addition to $\sigma_{v}^{2}$ and $\lambda_{v}$, the size of the feature vector is $\left|f_{v}\right|=\left|\alpha_{v}\right|+2$, i.e. 28 elements. Since the feature vector is constructed at each voxel $v$ in a given volume; this produces a four-dimensional (4D) parameter volume with dimensions $H \times W \times D \times\left|f_{v}\right|$ where $H, W, D$ are respectively the height, width and depth of the volume.

\section{Volumetric texture segmentation}

Our proposed method VTS utilizes the texture features of the volumetric image to assign a desired label to each voxel in a volume. We propose employing $k$-mean clustering to cluster the parameter volume into regions based on the volumetric texture features described by the feature vector $f_{v}$. However, before applying $k$-mean to the feature space, two issues related to the segmentation process need to be addressed. First, the idea of employing the estimation cube is to capture different texture patterns which means more discriminative features. However, when this cube is placed on the boundaries, it covers other voxels from different texture patterns surrounding the center of neighborhoods leading to poor segmentation performance. To overcome this problem, we propose a solution to maximize the discrimination between different patterns of texture. This is achieved by sliding an averaging volume $V_{m}$ with dimensions $m \times m \times m$ inside the parameter volume and assigns each $f_{v}$ new values. These new values are the average of the feature vectors of the surrounding voxels. This helps to increase the distance between region features in the feature space so that they are assigned different labels when $k$-mean is applied. The second issue regards isolated voxels, which appear either because of the presence of noise or due to the nature of texture structure in some regions of the image. These 


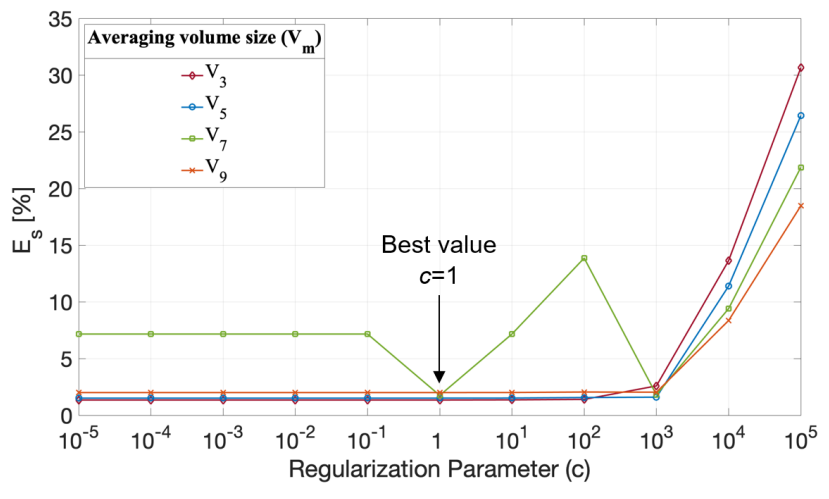

Figure 2. Segmentation error $E_{s}[\%]$ for different sizes of $V_{m}$ using multiple values of $c$.

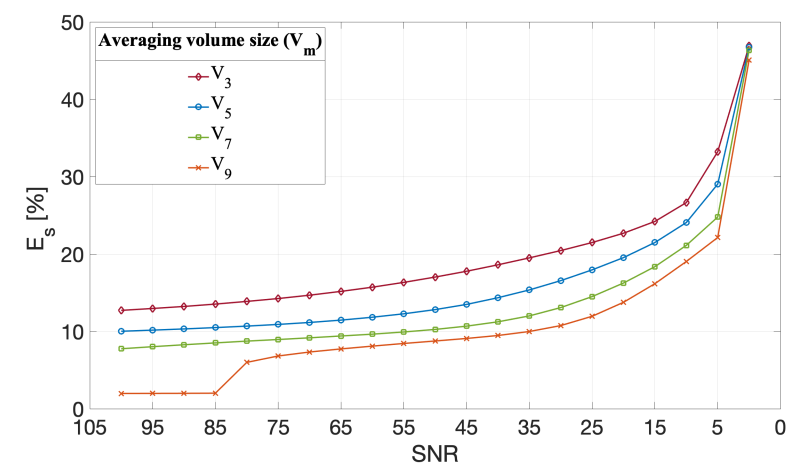

Figure 3. Segmentation error $E_{s}[\%]$ for different SNR values using different sizes of $V_{m}$ and $c=1$.

isolated voxels are effectively removed by using the solution of averaging volume proposed here. The details of VTS are illustrated in Fig 1.

\section{Method Evaluation}

Our proposed method is evaluated using the RFAI database [11]. This database contains different categories of volumetric textures for a performance evaluation of the classification and segmentation. As we are interested in the segmentation process, the synthetic volumetric texture samples with size $128 \times 128 \times 128$ specified for segmentation algorithm evaluation are selected from the database.

\section{A. Experimental setup}

In this experiment, two parameters are to be tuned to evaluate the performance of VTS. The first parameter is the regularization parameters $c$ (see equation 2) which is set to different values equal to $\left\{10^{-5}, \ldots, 10^{0}, \ldots, 10^{5}\right\}$ then the value that achieves the best performance over all samples is selected. The second parameter is the size of the averaging volume $V_{m}$ and also is set for different values of $\left\{V_{3}, V_{5}, V_{7}, V_{9}\right\}$ to better evaluate the performance of VTS. The neighborhood size is set by default to $n=3$
Table I

SEGMENTATIONS RESULTS REPRESENTED BY THE ERROR RATE $E_{s}[\%]$ FOR DIFFERENT SIZES OF $V_{m}$ AND $c=1$

\begin{tabular}{|c|c|c|c|}
\hline \multicolumn{4}{|c|}{ Averaging volume size $\left(V_{m}\right)$} \\
\hline$\left(V_{3}\right)$ & $\left(V_{5}\right)$ & $\left(V_{7}\right)$ & $\left(V_{9}\right)$ \\
\hline $\mathbf{1 . 3 4} \pm \mathbf{0 . 9 7}$ & $1.51 \pm 1.14$ & $1.75 \pm 1.40$ & $2.00 \pm 1.70$ \\
\hline
\end{tabular}

where the best value for the estimation cube size according to our experiment is $w=7$. This size of the estimation cube is sufficient to capture the underlying structure of texture while achieving the localization. The volumetric texture samples are assumed to be generated from two different classes of textures. Hence, the $k$-mean clustering algorithm is expected to cluster the volumetric textures into two separated volumes, i.e. $(k=2)$. Further experiments are conducted to evaluate the noise-resistance property of VTS. Different levels of Gaussian noise proportional to signalto-noise ratio (SNR) with values $(100,95,1)$ are added to volumetric texture samples. The SNR is calculated as a power ratio of the volumetric image over noise.

The segmentation performance is measured by the error rate $E_{s}$ and is calculated as the ratio between the number of incorrectly segmented voxels and the total number of voxels in the volume. The results are obtained by computing the mean and standard deviation of $E_{s}$ for each $V_{m}$ over all samples.

The segmentation results presented in Table 1 demonstrate the high performance of VTS for segmenting volumetric textures with minimum segmentation error $E_{s}$ of $1.34 \%$. The results are obtained for the best value of $c$, i.e. $c=1$ , selected in our experiments. It is observed from Fig 2 that the parameter $c$ has an influence on the segmentation performance of VTS which is more likely depending on the nature of textures. Nevertheless, the method performs well with all $V_{m}$ sizes at $c=1$. The effect of $V_{m}$ size differs slightly from sample to sample due the nature of structures underlying the texture, but relatively small $V_{m}$ tends to perform well. An example of VTS results of a selected sample illustrated in Fig 4 shows good segmentation of the textures forming the volume. The large $V_{m}$ (i.e. $V_{9}$ ) is beneficial in the presence of noise. Fig 3 indicates that larger $V_{m}$ tend to be less sensitive to noise due to the increase of parameter volume smoothing during the averaging process. As it can be noticed in Fig 3 with added noise up to SNR=50, VTS comparatively maintains a reduced segmentation error $E_{s}$ less than $9 \%$ when $V_{9}$ is used.

\section{B. Comparison to other method}

Our proposed method VTS is compared with M-VTS method [3] for volumetric texture segmentation. VTS is implemented on the same dataset generated in [3] and compared with the reported results of M-VTS . The results of the comparison evaluation presented in Table 2 show that 

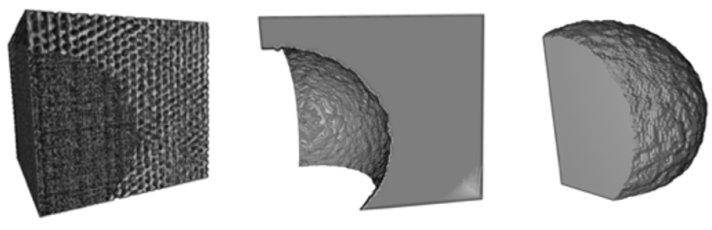

Figure 4. Example of the segmentation result using $V_{3}$.

VTS comparatively outperforms M-VTS on both obtained datasets in this experiment.

\section{CONCLUSION}

In this paper, a new method for volumetric texture segmentation, named VTS, is introduced. Based on 3D-GMRF model, the estimated parameters are employed as a feature vector to describe the local textures. These features are extracted for each voxel in the volume producing a parameter volume. Then the solution of averaging volume is proposed to refine the parameter volumes to remove isolated voxels and discriminate between different regions. The results of conducted experiments on different volumetric textures demonstrate excellent performance of VTS in extracting the discriminative features and segmenting the volumetric textures. VTS is robust to noise as reported in the experiment carried out by adding different levels of Gaussian noise. An experiment is also carried out to compare VTS with an existing method and the results show that VTS outperforms the method investigated in this paper.

\section{REFERENCES}

[1] S. Abbasi and F. Tajeripour. Detection of brain tumor in 3d mri images using local binary patterns and histogram orientation gradient. Neurocomputing, 219:526-535, 2017.

[2] H. Akbari and B. Fei. Automatic 3d segmentation of the kidney in $\mathrm{mr}$ images using wavelet feature extraction and probability shape model. In Medical Imaging 2012: Image Processing, volume 8314, page 83143D. International Society for Optics and Photonics, 2012.

[3] C. C. R. Aldasoro and A. Bhalerao. Volumetric texture segmentation by discriminant feature selection and multiresolution classification. IEEE Transactions on Medical Imaging, 26(1):1-14, 2007.

[4] Y. Almakady, S. Mahmoodi, J. Conway, and M. Bennett. Volumetric texture analysis based on three-dimensional gaussian markov random fields for copd detection. In Annual Conference on Medical Image Understanding and Analysis, pages 153-164. Springer, 2018.

[5] R. Chellappa and S. Chatterjee. Classification of textures using gaussian markov random fields. IEEE Transactions on Acoustics, Speech, and Signal Processing, 33(4):959-963, 1985.
Table II

COMPARISON RESULTS IN $E_{s}[\%]$.

\begin{tabular}{|c|c|cccc|}
\hline \multirow{2}{*}{ dataset } & \multicolumn{4}{|c|}{ method } \\
\cline { 2 - 6 } & M_VTS & \multicolumn{4}{|c|}{ Our method (VTS) } \\
\hline Gaussian & 6.2 & $V_{3}=1.5$ & $V_{5}=\mathbf{0 . 9}$ & $V_{7}=1.1$ & $V_{9}=1.3$ \\
\hline Oriented & 3.0 & $V_{3}=2.0$ & $V_{5}=1.2$ & $V_{7}=\mathbf{1 . 0}$ & $V_{9}=\mathbf{1 . 0}$ \\
\hline
\end{tabular}

[6] A. Depeursinge, A. Foncubierta-Rodriguez, D. Van De Ville, and $\mathrm{H}$. Müller. Three-dimensional solid texture analysis in biomedical imaging: review and opportunities. Medical image analysis, 18(1):176-196, 2014.

[7] C. Dharmagunawardhana, S. Mahmoodi, M. Bennett, and M. Niranjan. Gaussian markov random field based improved texture descriptor for image segmentation. Image and Vision Computing, 32(11):884-895, 2014.

[8] F. Hild, S. Roux, D. Bernard, G. Hauss, and M. Rebai. On the use of $3 \mathrm{~d}$ images and $3 \mathrm{~d}$ displacement measurements for the analysis of damage mechanisms in concrete-like materials. In VIII International Conference on Fracture Mechanics of Concrete and Concrete Structures FraMCoS-8, 2013.

[9] H. H. Ip and S. W. Lam. Using an octree-based rag in hyperirregular pyramid segmentation of texture volume. In Proc. of the IAPR Workshop on Machine Vision Applications. Citeseer, 1994.

[10] A. Madabhushi, M. Feldman, D. Metaxas, D. Chute, and J. Tomaszewski. A novel stochastic combination of $3 \mathrm{~d}$ texture features for automated segmentation of prostatic adenocarcinoma from high resolution mri. In International Conference on Medical Image Computing and Computer-Assisted Intervention, pages 581-591. Springer, 2003.

[11] L. Paulhac, P. Makris, J.-Y. Ramel, et al. A solid texture database for segmentation and classification experiments. In VISAPP (2), pages 135-141, 2009.

[12] M. Petrou and P. G. Sevilla. Image processing: dealing with texture, volume 1. Wiley Chichester, 2006.

[13] T. Randen, E. Monsen, C. Signer, A. Abrahamsen, J. O. Hansen, T. Sæter, and J. Schlaf. Three-dimensional texture attributes for seismic data analysis. In SEG Technical Program Expanded Abstracts 2000, pages 668-671. Society of Exploration Geophysicists, 2000.

[14] M. Tuceryan and A. K. Jain. Texture analysis. In Handbook of pattern recognition and computer vision, pages 235-276. World Scientific, 1993.

[15] U. Genschel and W. Q. Meeker. A comparison of maximum likelihood and median-rank regression for weibull estimation. Quality Engineering, 22(4):236-255, 2010.

[16] A. BJORKSTROM. Ridge regression and inverse problems. Stockholm University, Department of Mathematics 2001. 